\title{
ON THE TWO SHEETED COVERINGS OF CONICS BY ELLIPTIC CURVES
}

\author{
BY
}

R. E. MACRAE

\begin{abstract}
Let $K$ be the field of algebraic functions on an elliptic curve that can be described by an equation of the form $y^{2}=f(x)$ where $f(x)$ is a quartic polynomial over a field $k$. Moreover, assume that the Riemann surface for $K$ contains no points rational over $k$. When $k$ is the field of real numbers it is well known that $K$ may also be expressed as a quadratic extension of a function field $L=k(u, v)$ of algebraic functions on a conic whose Riemann surface also contains no points rational over $k$. We extend this result to $p$-adic ground fields $k$. Moreover, we describe the various subfields of index two and genus zero (conic subfields) in terms of the $k$-rational points on the Jacobian of $K$. This is done for arbitrary ground fields. In particular, the embedding of the projective class group of $K$ (over $k$ ) is seen to describe exactly those conic subfields that possess $k$-rational points.
\end{abstract}

1. Introduction. Function fields of genus one are very often given as quadratic extensions of function fields of genus zero. Geometrically this situation is that described in the title. In order for this to obtain it suffices that the elliptic curve (over $k$ ) have a $k$-rational or quadratic point. We consider, therefore, the following situation: (1) a field $k$ and a fixed quadratic extension $k_{1}$ are given, (2) a function field $K_{1}$ in one variable of genus. one over $k$ with a $k_{1}$-rational point but no $k$-rational point is given. We wish to describe the various subfields of index 2 and genus 0 in $K_{1}$ (conic subfields). We prove the following facts (among others): (1) There is an explicit one-to-one correspondence between the $k$-rational points, $J_{k}$, in the Jacobian variety and the conic subfields of $K_{1}$. (2) If $j\left(\bar{D}_{0}^{K}{ }_{1} / k\right)$ is the usual embedding of the class group of divisors of degree zero on $K_{1}$ into $J_{k}$, then a conic subfield corresponds to a point in $j\left(\bar{D}_{0}^{R_{1} / k}\right)$ iff that conic subfield has a $k$-rational point. (3) If $N J_{k_{1}}$ is the subgroup of norms of $k_{1}$-rational points on the Jacobian, then a conic subfield corresponds to a point in $N J_{k_{1}}$ iff the conic subfield has a $k$-rational point that lies under a quadratic point with coordinates in $k_{1}$. (4) $N J_{k_{1}} \leq j\left(\bar{D}_{0}^{K_{1} / k}\right)$ and (5) if $k$ is a $p$-adic field then $K_{1}$ contains a conic subfield without $k$-rational points. A variety of

Received by the editors April 1, 1974.

AMS (MOS) subject classifications (1970). Primary 14H05; Secondary 12B99. 
other information is derived-particularly in the case that $k$ is p-adic (see \$4). Moreover, it is shown, using the techniques developed, that the Jacobian of the so-called Reichardt equation $y^{2}=2\left(x^{4}-17\right)$ is given by $y^{2}=$ $x\left(x^{2}+17\right)$ over $Q$.

Several comments and a caution of a technical nature should be made at this point. A cursory reading of the succeeding sections of this paper may suggest that we are playing formal games with the standard cohomology of groups. This is, however, not the case as in analysis of the statements and scope of Theorem 3.7 and Theorem 4.6 will readily show. The technical caution concerns the perverse habit of the author to take his group action on the right and to compute his cocycles consistent with this convention. This makes the form of the equation for a 1-cocycle somewhat different from the standard one. However, the reader should have no difficulty in understanding the proofs.

The author wishes to express his gratitude to various persons and agencies that provided both intellectual and financial support during the period of time that this paper was written. In part this was the National Science Foundation and the University of Paris at Orsay for research grants. The University of Colorado provided me with a Faculty Fellowship during the academic year 1972-1973 that permitted me time to engage in research activities at the University of Paris. Finally I am greatly appreciative of many helpful conversations with P. Samuel and G. Poitou.

2. Preliminaries. We begin with a brief review of some known results simply in order to establish a convenient notation. Details may be found in [1]. We let $k$ be a completely arbitrary field and let $K$ be an elliptic function field over $k$. By this we mean that $K$ is a finitely, separably generated extension of $k$ such that $k$ is algebraically closed in $K$ and genus $\left(k_{1} \otimes_{k}\right.$ $\left.K / k_{1}\right)=1$ for all algebraic extensions $k_{1}$ of $k_{0}$. Now let $k_{1}$ be a Galois extension of $k$ and set $L=k_{1} \otimes_{k} K$. The following short exact sequence of Galois groups is easy to verify:

$$
1 \rightarrow \operatorname{Aut}\left(L / k_{1}\right) \rightarrow \operatorname{Aut}(L / k) \rightarrow \operatorname{Aut}\left(k_{1} / k\right) \rightarrow 1 .
$$

In the language of group extensions this is a split extension since Aut $(L / K)$ is a complete set of coset representatives that forms a group. By pairing each such group of representatives with the subfield it leaves pointwise fixed we establish a one-to-one correspondence between the various groups of representatives and the subfields $K_{1}$ of $L$ that contain $k$, are linearly disjoint from $k_{1}$ and such that $k_{1} K_{1}=L$.

Now let us assume further that $L$ has a $k_{1}$-rational point. We arbitrarily 
distinguish such a point and call it $\Re_{\infty}$. In the-usual way, the set $J_{k_{1}}$ of $k_{1}$-rational points of $L$ has the structure of an abelian group with $\Re_{\infty}$ as the neutral element. Indeed one sets up a correspondence between $J_{k_{1}}$ and the group $\bar{D}_{0}^{L / k_{1}}$ of divisor classes of degree zero. We now let $F\left(\oiint_{\infty}\right)$ consist of all automorphisms in Aut $\left(L / k_{1}\right)$ that leave $\beta_{\infty}$ fixed and $T\left(L / k_{1}\right)$ denote the subgroup of Aut $\left(L / k_{1}\right)$ consisting of translations. Now $T\left(L / k_{1}\right)$ is isomorphic to $J_{k_{1}}$ and is a normal subgroup of Aut $\left(L / k_{1}\right)$. Moreover Aut $\left(L / k_{1}\right)$ is the subdirect product of $T\left(L / k_{1}\right)$ and $F\left(\oiint_{\infty}\right)$ or, equivalently, the short exact sequence

$$
1 \rightarrow T\left(L / k_{1}\right) \rightarrow \operatorname{Aut}\left(L / k_{1}\right) \rightarrow \operatorname{Aut}\left(L / k_{1}\right) / T\left(L / k_{1}\right) \rightarrow 1
$$

is split and $F\left(\Re_{\infty}\right)$ is a group of representatives. All details may be found in [2, Chapter IV, $\$ 2]$.

Now let $\mathbb{B}_{1}=\left\{A_{\sigma} \mid \sigma \in\right.$ Aut $\left.\left(k_{1} / k\right)\right\}$ and $\mathbb{B}_{2}=\left\{B_{\sigma} \mid \sigma \in\right.$ Aut $\left.\left(k_{1} / k\right)\right\}$ be two groups of representatives with respect to the sequence (2.1). We assume that $A_{\sigma} \rightarrow \sigma$ and $B_{\sigma} \rightarrow \sigma$. It is always the case that $A_{\sigma}^{-1} B_{\sigma} \in$ Aut $\left(L / k_{1}\right)$ for all $\sigma \in$ Aut $\left(k_{1} / k\right)$.

Definition 2.2. $\mathbb{B}_{1}$ and $\mathbb{B}_{2}$ are said to be translation equivalent if $A_{\sigma}^{-1} B_{\sigma}$ is in $T\left(L / k_{1}\right)$ for all $\sigma$.

It is not difficult to see that this is in fact an equivalence relation. The following lemma follows immediately from various definitions while its corollary requires the remark that an automorphism is determined uniquely by its effect on prime divisors.

Lemma 2.3. Let $\Re_{1}, \Re_{2}$ be in $J_{k_{1}}$ and $A$ be in Aut $(L / k)$. Then (i) $\left(\Re_{1}+\Re_{2}\right)^{A}=\Re_{1}^{A}+\mathbb{P}_{2}^{A}-\mathbb{P}_{\infty}^{A}$ and (ii) $\left(-\Re_{1}\right)^{A}=-\Re_{1}^{A}+2 \mathbb{P}_{\infty}^{A}$.

Corollary 2.4. Let $T_{\beta}$ be the translation by the point $\$$ of $J_{k_{1}}$. Then $A^{-1} T_{\beta} A=T_{(\$} A_{\left.-\beta_{\infty}\right)}^{A}$, for every $A$ in $\operatorname{Aut}(L / k)$.

We will customarily write $T_{\S}^{A}=A^{-1} T_{\S} A$.

The following result is fundamental to the entire theory.

Theorem 2.5 (Chatalet). In each equivalence class of translation equiva. lent groups of representatives of (2.1) there is precisely one all of whose elements fix $\oiint_{\infty}$.

Proof. To show uniqueness we let $\mathbb{B}_{1}=\left\{A_{\sigma}, \ldots\right\}$ and $\mathbb{C}_{2}=\left\{B_{\sigma}, \ldots\right\}$ be two groups satisfying the conclusion of the theorem. Then $A_{\sigma}^{-1} B_{\sigma}=T_{\beta_{\sigma}}$

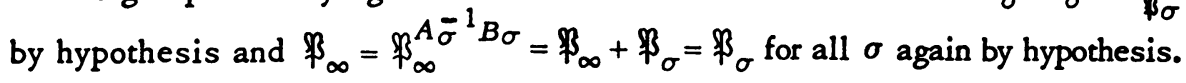


Thus $A_{\sigma}=B_{\sigma}$ for all $\sigma$. To show existence we let $\mathbb{B}_{1}=\left\{A_{\sigma}, \ldots\right\}$ be any member of a translation equivalent class. Let $B_{\sigma}=A_{\sigma} T{ }_{-\beta_{\infty}}^{A_{\sigma^{*}}}$ Since $\Re_{\infty}^{B \sigma}=$ $\beta_{\infty}$ for all $\sigma$, it suffices to show that $\mathbb{B}_{2}=\left\{B_{\sigma} \ldots\right\}$ is in fact a group. But by Corollaries 2.3 and 2.4 we see that

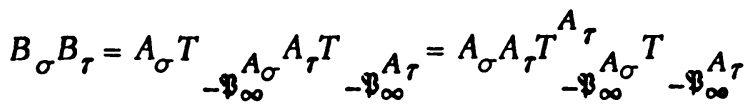

$$
\begin{aligned}
& =A_{\sigma \tau} T_{\left(-\beta_{\infty}\right)^{A_{\sigma}} A_{\tau}-2 \beta_{\infty}^{A_{\tau}}}=A_{\sigma \tau} T_{-\wp_{\infty}}^{A_{\sigma} A_{\tau}}=B_{\sigma \tau}
\end{aligned}
$$

Also $B_{\sigma} B_{\sigma-1}=A_{1}$ so $B_{2}$ is closed under inverses as well.

From now on we will assume a class of translation equivalent groups has been selected and that $\mathbb{N}_{0}$ is the unique member whose elements fix $\beta_{\infty}$. By an abuse of notation the elements of $\mathbb{C}_{0}$ will be denoted by $\sigma, r, \ldots$ as are the elements of Aut $\left(k_{1} / k\right)$. We regard $T\left(L / k_{1}\right)$ as a right $A u t\left(k_{1} / k\right)-$ module by utilizing its natural structure as a right $\mathbb{A}_{0}$-module. We have

Theorem 2.6. There is a one-to-one correspondence between the members of the translation equivalent class and the cocycles of $Z^{1}\left(\right.$ Aut $\left.\left(k_{1} / k\right) ; T\left(L / k_{1}\right)\right)$ and $f_{0}$ corresponds to the trivial cocycle.

The proof is immediate.

We now consider the various subfields $K_{0}, K_{1}, \ldots$ left pointwise fixed by the groups $\mathbb{P}_{0}, \mathbb{B}_{1}, \ldots$ in a translation equivalent class. As we know, each is an elliptic function field over $k$.

Definition 2.7. We say that two of these fields $K_{i}, K_{j}$ are $k_{1}$-translates if there is a translation $T_{\beta}$ in $T\left(L / k_{1}\right)$ such that $K_{i}^{T_{\beta}^{i}}=K_{j}$.

This is clearly an equivalence relation.

Theorem 28. $K_{i}$ is a $k_{1}$-translate of $K_{0}$ if and only if $K_{i}$ has a $k$ rational point.

Proof. Let $B_{i}=\left\{\sigma T_{\beta_{\sigma}}, \ldots\right\}$ be the group associated with $K_{i}$ and $\left\{T_{\beta_{\sigma}}, \ldots\right\}$ the associated cocycle. Clearly $K_{i}$ has a $k$-rational point if and only if there is a $k_{1}$-rational point $\Re$ in $L$ such that $\Re^{\sigma T} \beta_{\sigma}=\Re$. That is,

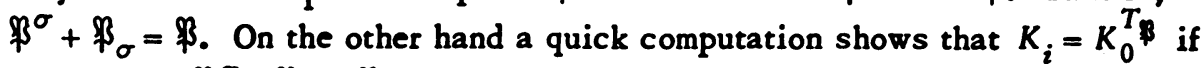
and only if $\Re^{\sigma}+\Re_{\sigma}=\Re_{\text {. }}$.

Another way of looking at Theorem 2.8 is

Theorem 2.9. $K_{i}$ is a $k_{1}$-translate of $K_{j}$ if and only if their associated cocycles differ by a coboundary in $B^{1}\left(A u t\left(k_{1} / k\right) ; T\left(L / k_{1}\right)\right)$. Thus there is a one-to-one correspondence between the elements of $H^{1}\left(\right.$ Aut $\left.\left(k_{1} / k\right) ; T\left(L / k_{1}\right)\right)$ 
and the equivalence classes of $k_{1}$-translates with the trivial member of $H^{1}$ being associated with the class of $K_{0^{\circ}}$.

We remark at this point that in dealing with the various group cohomologies we will use $T\left(L / k_{1}\right)$ and $J_{k_{1}}$ interchangeably with no further warning.

Now let $D_{0}^{K_{i} / k}$ be the group of divisors of degree zero for $K_{i}$ In an obvious way there is a homomorphism of $D_{0}^{K_{i} / k}$ into $D_{0}^{L / k_{1}}$ and hence into $J_{k_{1}}=\bar{D}_{0}^{L / k_{1}}$, the group of divisor classes of degree zero. Now it is an easy consequence of "Hilbert's Theorem 90" that the kernel of this homomorphism is the group of principal divisors of $K_{i}$. Hence we have a monomorphism $j: \bar{D}_{0}^{K_{i}{ }^{\gamma_{k}}} \rightarrow J_{k_{1}}$.

Theorem 2.10. $j\left(\bar{D}_{0}^{K_{i} / k}\right) \leq J_{k^{\circ}}$

Proof. It is clear that $J_{k}=\left\{\Re \in J_{k_{1}} \mid \Re^{\sigma}=\Re\right.$ for all $\left.\sigma\right\}$. Now if $\Re \epsilon$ $j\left(\bar{D}_{0}^{K_{i} / k}\right)$ there is a divisor $\mathcal{Q}$ of degree zero on $K_{i}$ such that $\mathcal{U}$ is linearly

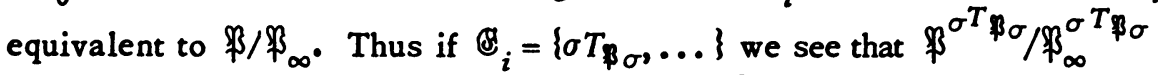
is linearly equivalent to $\Re / \mathbb{P}_{\infty}$. That is $\mathbb{\beta}=\mathbb{\beta}^{\sigma}$.

3. Conic subfields. Our notation and conventions remain as in $\$ 2$ except that $k_{1}$ will now be a fixed quadratic extension of $k$ and Aut $\left(k_{1} / k\right)$ $=\{1, \sigma\}_{0}$

Definition 3.1. A subfield $K_{i 1}$ of $K_{i}$ is said to be conic if (i) $k \leq K_{i 1}$, (ii) genus $\left(K_{i 1} / k\right)=0$ and (iii) $\left[K_{i}: K_{i 1}\right]=2$.

Theorem 3.2. There is a one-to-one correspondence between the conic subfields of $K_{i}$ and the points of $J_{k}$.

Proof. Since $L$ has the $k_{1}$-rational point $\bigotimes_{\infty}$ there is a one-to-one correspondence between the conic subfields of $L$ and the points of $J_{k_{1}}$ given by associating the point $\Re$ to the fixed field of the automorphism $\mu T_{\sharp}$ where $\mu$ is the reflexion automorphism (i.e., $\mathbb{P}^{\mu}=-\$$ ). Now $K_{i}$ is the fixed field of $\sigma T_{\beta \sigma}$ Thus if $\sigma T_{\beta \sigma}$ and $\mu T_{\S}$ commute they generate a group of order 4 and the fixed field of this group is clearly a conic subfield of $K_{i}$. Conversely if $K_{i 1}$ is a conic subfield of $K_{i}$ then $k_{1} K_{i 1}$ is a conic subfield of $L$ and the point $¥$ is associated and $\mu T_{\Re}$ and $\sigma T_{\S \sigma}$ commute. Finally $\mu T_{\Re} \sigma T_{\Re \sigma}=$ $\sigma T_{\beta} \mu T_{\beta}$ if and only if $\mu \sigma T_{\beta^{\sigma}+\beta_{\sigma}}=\sigma \mu T_{\beta-\beta_{\sigma}}$ if and only if $\beta^{\sigma}+\Re_{\sigma}=\beta-$

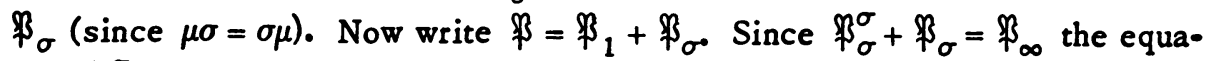
tion $\Re^{\sigma}+\Re_{\sigma}=\Re_{-} \Re_{\sigma}$ translates into $\Re_{1}^{\sigma}=\Re_{1}$. That is to say $\Re_{1} \in J_{k^{\circ}}$. 
Definition 3.3. Let $K_{i m}$ and $K_{i n}$ be conic subfields of $K_{i}$ associated with the points $\bigotimes_{m}$ and $\bigotimes_{n}$, respectively, of $J_{k}$. We say that $K_{i m}$ is a $k_{1}$ translate of $K_{i n}$ if there is a $k_{1}$-translate $K_{j}=K_{i}^{T}$ of $K_{i}$ such that the conic subfield $K_{i n}^{T ß}$ is associated with the point $\oiint_{m}$.

In order to see that this relation is in fact an equivalence relation it suffices to verify the following proposition.

Proposition 3.4. $K_{\text {im }}$ is a $k_{1}$-translate of $K_{\text {in }}$ if and only if $\Re_{m}=\Re_{n}+$ $\Re+\Re^{\sigma}$ where $\Re$ is some point in $J_{k_{1}}$.

Proof. We note that if $\bigotimes_{\sigma}$ is the cocycle associated with $K_{i}$ then $\beta_{\sigma}+$ $\Re-\Re^{\sigma}$ is the cocycle associated with $K_{j}=K_{i}^{T}$. Likewise the point of $J_{k}$ associated with $K_{i n}^{T}$ is $\bigotimes_{n}+\Re^{\top} \Re^{\sigma}$.

We thus have an interpretation of $H^{2}\left(A u t\left(k_{1} / k\right) ; J_{k_{1}}\right)$.

Theorem 3.5. There is a one-to-one correspondence between the equivalence classes of $k_{1}$-translate conic subfields of $K_{i}$ and the elements of $H^{2}\left(\operatorname{Aut}\left(k_{1} / k\right) ; J_{k_{1}}\right)$.

Proof. Since $k_{1}$ is a cyclic extension of $k$ we have $H^{2}\left(A u t\left(k_{1} / k\right) ; J_{k_{1}}\right)$ $=J_{k} / N J_{k_{1}}$ where $N J_{k_{1}}=\left\{\Re+\Re^{\sigma} \mid \Re \in J_{k_{1}}\right\}$. Now apply Proposition 3.4.

We also have an interpretation of the trivial class.

Theorem 3.6. Let $K_{i 1}$ be the conic subfield of $K_{i}$ associated with the point $\Re_{1}$. Moreover assume $K_{i}$ is not itself in the trivial class. Then $\Re_{1} \epsilon$ $N J_{k_{1}}$ if and only if $K_{i 1}$ has a k-rational point that lies under a quadratic point of $K_{i}$ that splits in $L$.

Proof. Suppose $\Re_{1}=\Re_{+} \Re^{\sigma}$. Since $\Re_{\sigma}$ is not the trivial cocycle, the points $\Re$ and $\Re^{\sigma T_{\mathfrak{F}}}=\Re^{\sigma}+\Re_{\sigma}$ are distinct. Thus they lie over a quadratic point of $K_{i}$ that splits in $L$. On the other hand, $\Re^{\mu T_{\mathfrak{B}+} \Re+\Re_{\sigma}}=\Re^{\sigma}+\Re_{\sigma}=$ $\oiint^{\sigma T \beta \sigma}$ so this quadratic point lies over a k-rational point of $K_{i 1}$. This argument can be reversed in order to prove the converse.

Perhaps the most interesting interpretation is contained in the following result.

Theorem 3.7. Let $K_{i 1}$ be the conic subfield associated with the point $\Re_{1}$. Then $\Re_{1} \in j\left(\bar{D}_{0}^{K_{i} / k}\right)$ if and only if $K_{i 1}$ has a k-rational point.

Proof. First of all let us parametrize $K_{0}$ by a nonsingular plane cubic so that $K_{0}=k(x, y)$ and $L=k_{1}(x, y)$. Now $k_{1} K_{i 1}$ is the fixed field of 


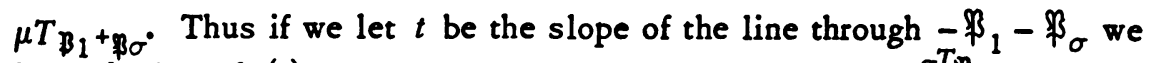
have $k_{1} K_{i 1}=k_{1}(t)$. Since $\sigma T_{B_{\sigma}}$ and $\mu T_{B_{1}+\$_{\sigma}}$ commute, $t^{\sigma T_{B_{\sigma}}}=$ $(a t+b) /(c t+d)$ for suitable $a, b, c, d$ in $k_{1}$ with $a d-b c \neq 0$. Now let the $x$ and $y$ coordinates of $-\$_{1}-\bigotimes_{\sigma}$ be $\alpha$ and $\beta$, respectively. Then $t=$ $(y-\beta) /(x-\alpha)$. Thus $t^{\sigma T_{\mathfrak{B} \sigma}}=\left(y^{T_{\mathfrak{B} \sigma}}-\beta^{\sigma}\right) /\left(x^{T_{\mathfrak{B} \sigma}}-\alpha^{\sigma}\right)$ (recall that $y^{\sigma}=$

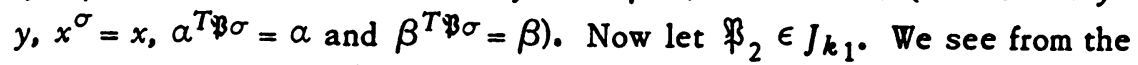
above remarks that $t\left(\oiint_{2}\right)$ is the slope of the line through $\sharp_{2}$ and $-\sharp_{1}-\sharp_{\sigma}$

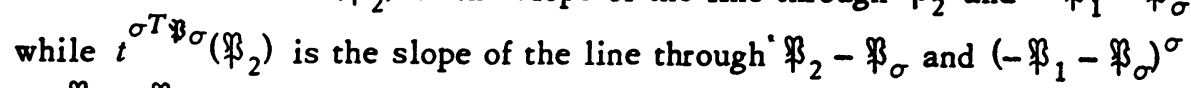
$=-\Re_{1}+\Re_{\sigma}$. We distinguish two cases. Case 1: $c=0$. We claim that this occurs if and only if $\Re_{1}=\Re_{\infty}$. Indeed $t\left(\Re_{\infty}\right)=\infty$ so $c=0$ if and only if $t^{\sigma T} \Re_{\sigma}\left(\Re_{\infty}\right)=\infty$. Since the latter is the slope of the line through $\Re_{\infty}-\Re_{\sigma}=$ $-\sharp_{\sigma}$ and $-\sharp_{1}+\Re_{\sigma}$, we see that the third point on the line must be $\Re_{\infty}$ (note that $\Re_{\sigma} \neq \Re_{\infty}$ since $\Re_{\sigma}$ is assumed nontrivial). Thus $c=0$ if and only if $\Re_{1}=\Re_{\infty}$. Continuing with this case, it suffices to set $d=1$ so $t^{\sigma T^{T} \sigma}=a t+$ b. We claim $a=-1$ and $b=0$. To see this we let $\bar{k}$ be the separable closure of $k$ and extend $\sigma$ to an automorphism of $\bar{k}$ over $k$ in any of the possible ways. We extend $T_{B_{\sigma}}$ to $\bar{L}=\bar{k} \otimes_{k_{1}} L$ in the obvious way as the translation by $\Re_{\sigma}$. We see that if $\Re_{2}$ is any point in $J_{-}$then $t\left(\Re_{2}\right)$ is the slope

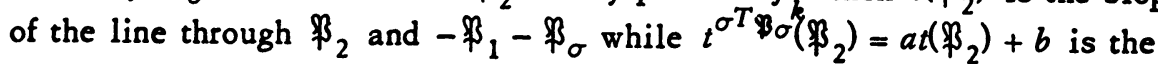

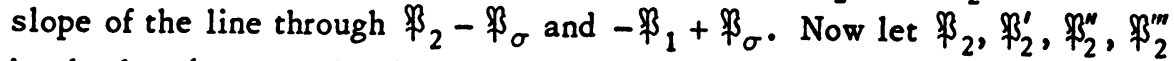
be the four (necessarily distinct) points of tangency to the line through $-\S_{\sigma}$. Moreover let $t_{2}=t\left(\oiint_{2}\right), \ldots, t_{2}^{\prime \prime \prime}=t\left(\Re_{2}^{\prime \prime \prime}\right)$. Since $\sharp_{2}-\oiint_{\sigma}=-\Re_{2}$ we have $-t_{2}=a t_{2}+b, \ldots,-t_{2}^{\prime \prime \prime}=a t_{2}^{\prime \prime \prime}+b$. Since these slopes are distinct, one must have $a=-1, b=0$. Finally let $k_{1}=k\left(\sqrt{d}_{0}\right)$ and $t^{\prime}=\sqrt{d_{0}} t_{0}$ Clearly $k_{1}(t)$ $=k_{1}\left(t^{\prime}\right)$ while $\left(t^{\prime}\right)^{\sigma T_{\sigma}}=t^{\prime}$ implies that $k\left(t^{\prime}\right) \leq K_{i 1}$. But the containment cannot be proper since. $k_{1}\left(t^{\prime}\right)=k_{1} K_{i 1}$. Hence $K_{i 1}$ has a $k$-rational point. Case 2: $c \neq 0$. It is no loss of generality to take $c=1$ so $t^{\sigma{ }^{\top} \sigma}=$ $(a t+b) /(t+d)$. Now $\left(\sigma T_{\mathfrak{B} \sigma}\right)^{2}=1$ so

$$
t=\left(\left(a a^{\sigma}+b^{\sigma}\right) t+\left(a^{\sigma} b+b^{\sigma} d\right)\right) /\left(\left(a+d^{\sigma}\right) t+\left(b+d^{\sigma} d\right)\right) .
$$

Thus $a a^{\sigma}+b^{\sigma}=b+d^{\sigma} d \neq 0$ while $a^{\sigma} b+b^{\sigma} d=a+d^{\sigma}=0$. Hence $d=-a^{\sigma}$ and $b=b^{\sigma}$. Let $\Delta=a a^{\sigma}+b$ and note that $\Delta \epsilon k^{*}$. Moreover $\left(t-a^{\sigma}\right)^{\sigma T_{B}}$ $=\Delta /\left(t-a^{\sigma}\right)$. Again let $k_{1}=k\left(\sqrt{d_{0}}\right)$ and write $t-a^{\sigma}=u+\sqrt{d_{0}} v$ with $u_{\text {o }}$ $v \in K_{i 1}$. Thus

$$
\Delta=\left(t-a^{\sigma}\right)\left(t-a^{\sigma}\right)^{\sigma^{T} B_{\sigma}}=\left(u+\sqrt{d_{0}} v\right)\left(u-\sqrt{d_{0}} v\right)=u^{2}-d_{0} v^{2} .
$$

Since $k_{1}\left(t-a^{\sigma}\right)=k_{1}(u, v)=k_{1} K_{i 1}$ we have $K_{i 1}=k(u, v)$. Consequently $K_{i 1}$ has a $k$-rational point if and only if $u^{2}-d_{0} v^{2}=\Delta$ has a solution in $k_{\text {. }}$ In other words, if and only if $\Delta \epsilon N k_{1}^{*}$. Let us look now at the principal 
divisor $\left(t-a^{\sigma}\right)$. We have $\left(t-a^{\sigma}\right)=\Re_{1} \Re_{\sigma} / \Re_{\infty}\left(\Re_{1}+\Re_{\sigma}\right)$. Indeed, the poles of $t^{\sigma T_{\beta}}$ are exactly the zeros of $t-a^{\sigma}$. Hence $\left(t-a^{\sigma}\right)=\left(\Re_{1} / \Re_{\infty}\right)^{1-\sigma T_{\beta}}$. From this equation it follows easily that $\Delta \epsilon N k_{1}^{*}$ if and only if there is an element $\omega$ in $L^{*}$ such that $\left(\omega \beta_{1} / \mathbb{\beta}_{\infty}\right)=\left(\omega \mathbb{\beta}_{1} / \Re_{\infty}\right)^{\sigma T} \beta_{\sigma}$. In other words $\Re_{1}$ $\epsilon j\left(\bar{D}_{0}^{K}{ }^{\prime / k}\right)$.

Corollary 3.8. $N J_{k_{1}} \leq j\left(\bar{D}_{0}^{K_{i 1 / k}}\right)$.

4. The local case. The results of this section will mostly pertain to the case in which $k$ is a p-adic field. We begin, however, with a construction which is general. Our notation and assumptions remain as in $\$ 3$.

We let $K_{0}^{\prime}$ be the fixed field for the involution $\sigma \mu$. It is easy to see that $K_{0}^{\prime}$ is an elliptic extension of $k$ with $\oiint_{\infty}$ as a $k$-rational point and $k_{1} K_{0}^{\prime}=$ $L$. Let $J_{k}^{\prime}$ denote the group of $k$-rational points of $K_{0}^{\prime} .\left(\oiint_{\infty}\right.$. is the neutral element.) Moreover, we will write $J_{k_{1}}^{\prime}$ instead of $J_{k_{1}}$ when we regard $L$ as $k_{1} K_{0}^{\prime}$. Now Aut $\left(k_{1} / k\right)$ acts on $J_{k_{1}}^{\prime}$ by $\Re_{\beta} \Re^{\sigma \mu}$. Thus we obtain the following duality relations:

$$
J_{k}=Z^{1}\left(\operatorname{Aut}\left(k_{1} / k\right) ; J_{k_{1}}^{\prime}\right) \text { and } J_{k}^{\prime}=Z^{1}\left(\operatorname{Aut}\left(k_{1} / k\right) ; J_{k_{1}}\right) \text {. }
$$

Next let us denote the Tate-Shafaravich maps reduced to the cyclic layer determined by $k_{1}$ as follows:

and

$$
\phi: J_{k} \times Z^{1}\left(\operatorname{Aut}\left(k_{1} / k\right) ; J_{k_{1}}\right) \rightarrow H^{2}\left(\operatorname{Aut}\left(k_{1} / k\right) ; k_{1}^{*}\right)
$$

$$
\phi^{\prime}: Z^{1}\left(\operatorname{Aut}\left(k_{1} / k\right) ; J_{k_{1}}^{\prime}\right) \times J_{k}^{\prime} \rightarrow H^{2}\left(\operatorname{Aut}\left(k_{1} / k\right) ; k_{1}^{*}\right) \text {. }
$$

See [4] for explicit details of the pairing.

Proposition 4.1. Let $\aleph_{1}$ be in $J_{k}$ and $\Re_{\sigma}$ be in $Z^{1}\left(\operatorname{Aut}\left(k_{1} / k\right) ; J_{k_{1}}\right)$. Then $\phi\left(\Re_{1}, \Re_{\sigma}\right)=\phi^{\prime}\left(\Re_{1}, \Re_{\sigma}\right)$.

Proof. Let $\rho$ be a generator for the fixed field of $\mu T_{\beta_{1}}+\beta_{\sigma}$ such that

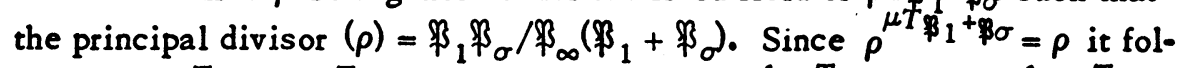

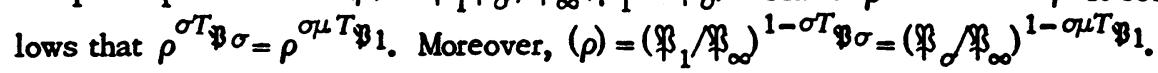
Hence, $\phi\left(\Re_{1}, \Re_{\sigma}\right)=\rho^{1+\sigma T_{\mathfrak{O}_{N}}^{*}}=\phi^{\prime}\left(\Re_{1}, \Re_{\sigma}\right)$.

From now on, $k$ will be a $p$-adic field. We assume known the exactness of the Tate-Shafaravich pairing. See [1], for example. Using this fact and Proposition 4.1 we obtain with no difficulty the following

Corollary 4.2. The pairing induces a nonsingular pairing 
$\bar{\phi}: H^{2}\left(\operatorname{Aut}\left(k_{1} / k\right) ; J_{k_{1}}\right) \times H^{1}\left(\operatorname{Aut}\left(k_{1} / k\right) ; J_{k_{1}}\right) \rightarrow H^{2}\left(\operatorname{Aut}\left(k_{1} / k\right) ; k_{1}^{*}\right)$.

The next three corollaries depend on the fact that when $k$ is $p$-adic then $J_{k}$ contains a subgroup $\mathfrak{D}$ isomorphic to the additive group of integer's of $\boldsymbol{k}$ and of finite index in $J_{k}$. See [1, Theorem 17.1].

Corollary 4.3. $H^{1}\left(\operatorname{Aut}\left(k_{1} / k\right) ; J_{k_{1}}\right) \cong H^{2}\left(\operatorname{Aut}\left(k_{1} / k\right) ; J_{k_{1}}\right)$

Proof. It suffices to show that either of these groups is finite. But $J_{k} / 2 J_{k} \rightarrow H^{2}\left(\right.$ Aut $\left.\left(k_{1} / k\right) ; J_{k_{1}}\right) \rightarrow 0$ and $J_{k} / 2 J_{k}$ is finite since $2 \mathfrak{D}$ is of finite index in $\mathscr{D}$ for all primes $p$.

Corollary 4.4. If $p \neq 2$ then the order of $H^{1}\left(A u t\left(k_{1} / k\right) ; J_{k_{1}}\right)$ is 1,2 or 4 .

Proof. Let $J_{k, 2}$ be the 2-primary component of $J_{k}$ and $\left(J_{k} / \mathscr{D}\right)_{2}$ the 2primary component of $J_{k} / \mathcal{D}$. It is easily seen that $J_{k, 2} \rightarrow\left(J_{k} / \mathscr{D}\right)_{2} \rightarrow 0$. Next, $\left(J_{k} / \mathscr{D}\right)_{2} \rightarrow J_{k} / 2 J_{k} \rightarrow 0$ since $\mathfrak{D}=2 \mathfrak{D}$. The proof is completed by observing that $J_{k} / 2 J_{k} \rightarrow H^{2}\left(\right.$ Aut $\left.\left(k_{1} / k\right) ; J_{k_{1}}\right) \rightarrow 0$.

Corollary 4.5. If $p \neq 2$ and $K_{0}$ is given by $y^{2}=P_{3}(x)$ with $P_{3}(x)$ an irreducible cubic, then $H^{1}\left(\right.$ Aut $\left.\left(k_{1} / k\right) ; J_{k_{1}}\right)=0$.

Proof. $J_{k, 2}=0$.

Our final result is an easy consequence of Theorem 3.7 and Proposition 4.1 and Corollary 4.2.

Theorem 4.6. If $K_{1}$ is an elliptic function field without rational points over the p-adic field $k_{\text {, then }} K_{1}$ contains a conic subfield $K_{11}$ without $k$ rational points.

5. Special computations. We begin with the general cubic $y^{2}=x^{3}+$ $A x^{2}+B x+C$ over the field $k$ (characteristic not 2). Set $k_{1}=k(\sqrt{d}$ ). Let $\Re_{\sigma}=(\alpha, \beta) \neq \Re_{\infty}$ be an arbitrary nonzero cocycle. In other words $\Re_{\infty}=\Re_{\sigma}+$ $\mathfrak{P}_{\sigma}^{\sigma}$ and so $\alpha \in k$ and $\beta^{\sigma}=-\beta$. Let $t=(y+\beta) /(x-\alpha)$ be the slope of the line through $-\mathbb{\beta}_{\sigma}=-\Re_{\infty}-\Re_{\sigma}$. As we have seen, $t^{\sigma^{T} \Re_{\sigma}}=-t$. Now $y^{2}=$ $t^{2}(x-\alpha)^{2}-2 \beta t(x-\alpha)+\beta^{2}$ implies $t^{2}(x-\alpha)-2 \beta t=x^{2}+\alpha x+\alpha^{2}+$ $A(x+\alpha)+B$ or $x^{2}+\left(\alpha+A-t^{2}\right) x+\left(\alpha^{2}+\alpha A+B+\alpha t^{2}+2 \beta t\right)=0$. Hence $x=\left[-\left(\alpha+A-t^{2}\right)+\omega\right] / 2$ where $\omega^{2}=\left(\alpha+A-t^{2}\right)^{2}-4\left(\alpha^{2}+\alpha A+B+a t^{2}+2 \beta t\right)$. Since $t^{\sigma T \sigma}=-t, \beta^{\sigma^{T_{\boldsymbol{B}}}}=\beta^{\sigma}=-\beta$ and $\alpha^{\sigma^{\top T_{\boldsymbol{B}}}}=\alpha^{\sigma}=\alpha$, it follows that $\omega^{\sigma T^{T} \sigma}= \pm \sigma$. We claim that the minus sign prevails. Indeed if $\omega^{\sigma T^{T} \sigma}=\omega$

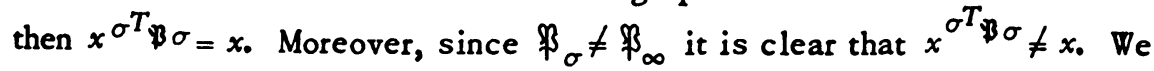
have therefore shown 
Proposition 5.1. With the notation as above, a quartic equation for the principal homogeneous space, $K_{1}$, associated with $\Re_{\sigma}$ is given by $d \bar{\omega}^{2}=$ $\left(\alpha+A-d \bar{t}^{2}\right)^{2}-4\left(\alpha^{2}+\alpha A+B+\alpha d \bar{t}^{2}+2 \beta \sqrt{d} t\right)$.

Let us now consider several special cases. Let $y^{2}=x\left(x^{2}+b x+c\right)$ and $\oiint_{\sigma}=(0,0)$. Specializing Proposition 5.1 we see that $K_{1}$ is given by $d \omega^{2}=$ $d^{2} \bar{t}^{4}-2 b d \bar{t}^{2}+b^{2}-4 c$. Next set $\oiint_{1}=(0,0)$ and let us compute the conic subfield $K_{11}$. The slope of the line through $-\Re_{1}-\Re_{\sigma}=\Re_{\infty}$ is the variable $x_{\text {. Since }}$ the slope of the line through $\beta_{1}$ and $\beta_{\sigma}$ is 0 in this case it follows that $x^{\sigma^{T} \Re_{\sigma}}=\Delta / x_{0}$. Now if $\Re_{2}=1 / 2 \Re_{\sigma}$ we know that $\Re_{2}^{\sigma T_{\sigma}}=-\Re_{2}$. Thus if $\Re_{2}=$ $\left(x_{2}, y_{2}\right)$ we see that $\Delta=x_{2}^{2}$. To compute $\Re_{2}$ let $r=y / x$ be the slope through $\Re_{\sigma}$. Hence $y^{2}=r^{2} x^{2}=x\left(x^{2}+b x+c\right)$ or $x^{2}+\left(b-r^{2}\right) x+c=0$. Thus $x_{2}=$ $\left[-\left(b-r_{2}^{2}\right) / 2\right]$ where $r_{2}^{4}-2 b r_{2}^{2}+b^{2}-4 c=0$. Now $r_{2}^{2}=b \pm 2 \sqrt{c}$ so $x_{2}= \pm \sqrt{c}$. We have proved

Proposition 5.2. With $\Re_{1}$ and $\Re_{\sigma}$ as above we have $\phi\left(\Re_{1}, \Re_{\sigma}\right)=c N k_{1}^{*}$.

Continuing, we set $x=u+\sqrt{d} v$ with $u, v$ in $K_{11}$. Then $K_{1}=k(u, v)$ and $u^{2}-d v^{2}=c$. Moreover, since $t^{2}=(y / x)^{2}=(x+(c / x)+b)=2 u+b$ and $t^{\sigma T_{\mathfrak{B} \sigma}}=-t$, we have $\overline{d t}^{2}=2 u+b$ where $\sqrt{d} \bar{t}=t$. Thus we have

Proposition 5.3. With all notation as above, $K_{11}$ is given by the equation $u^{2}-d v^{2}=c$ and $K_{1}$ is given by the pair of equations $u^{2}-d v^{2}=c$ and $d \bar{t}^{2}=2 u+b$.

It is, perhaps, of interest to specialize all of this for the equation $y^{2}=$ $x\left(x^{2}+17\right)$ over $k=Q$. We let $k_{1}=Q(\sqrt{2})$ and $\beta_{\sigma}=(0,0)$. Then the equation for the principal homogeneous space is given by $\bar{\omega}^{2}=2\left(\bar{t}^{4}-17\right)$. This latter equation is well known as the so-called Reichardt equation and is an example of an elliptic curve defined over $Q$ which has no rational points but which has points in every completion of $Q$. See $[1, \$ 26]$ for details. We have

Proposition 5.4. The equation for the Jacobian variety of the Reichardt equation $y^{2}=2\left(x^{4}-17\right)$ over $Q$ is $y^{2}=x\left(x^{2}+17\right)$.

Finally we consider the case of a cubic of the form $y^{2}=x(x-a)(x-c)$ and $\bigotimes_{\sigma}=(0,0)$ and $\bigotimes_{1}=(a, 0)$. Clearly $-\bigotimes_{1}-\bigotimes_{\sigma}=(c, 0)$. Let $t=y /(x-c)$ be the slope of the line through $-\S_{1}-\mathbb{\beta}_{\sigma}$. Thus $y^{2}=t^{2}(x-c)^{2}=x(x-a)$. $(x-c)$ or $x^{2}-\left(z+t^{2}\right) x+c t^{2}=0$. Hence $x=\left[\left(a+t^{2}\right)+\omega\right] / 2$ where $\omega^{2}=$ $t^{4}+2(a-2 c) t^{2}+a^{2}$. Now since the slope of the line through $\Re_{1}$ and $\Re_{\sigma}$ is zero we know that $t^{\sigma T_{\mathfrak{B}} \sigma}=\Delta / t$. In order to compute $\Delta$ we first find $1 / 2 \mathbb{P}_{\sigma}$ 
Indeed let $r=y / x$ be the slope of the line through $\Re_{\sigma}$. We have $y^{2}=r^{2} x^{2}=$ $x(x-a)(x-c)$ so $x^{2}-\left(a+c+r^{2}\right) x+a c=0$. Hence if $1 / 2 \beta_{\sigma}=\left(x_{2}, y_{2}\right)$ we have $x_{2}=\left(a+c+r_{2}^{2}\right) / 2$ where $0=\left(a+c+r_{2}^{2}\right)^{2}-4 a c=r_{2}^{4}+2(a+c) r_{2}^{2}+$ $(a-c)^{2}$. In other words, $r_{2}^{2}=-(a+c) \pm 2 \sqrt{a c}$. Hence $x_{2}= \pm \sqrt{a c}$ and $y_{2}$ $=x_{2} 2_{2}$. Since $\left(1 / 2 \beta_{\sigma}\right)^{\sigma^{T} \mathbb{P}_{\sigma}}=-\left(1 / 2 \Re_{\sigma}\right)$ it follows that $-y_{2} /\left(x_{2}-c\right)=$ $\Delta /\left(y_{2} /\left(x_{2}-c\right)\right)$ so $\Delta=-y_{2}^{2} /\left(x_{2}-c\right)^{2}=a$. Now set $t=u+\sqrt{d} v$ so that $u^{2}$ $-d v^{2}=a$ is the equation for the conic subfield $K_{11}$. Finally $(\omega / t)^{2}=t^{2}+$ $a^{2} / t^{2}+2(a-2 c)$ or $(\omega / t)^{2}=2\left(u^{2}+d v^{2}\right)+2(a-2 c)$. Hence $(\omega / t)^{\sigma T}{ }= \pm$ $(\omega / t)$. We claim that the minus sign prevails. Indeed if $(\omega / t)^{\sigma^{\top} T \sigma}=(\omega / t)$ it follows that $(x / t)=u+(\omega / 2 t)$ is fixed under $\sigma T_{\mathfrak{B} \sigma}$ But $(x / t)^{\sigma T \sigma=}$ $x^{\sigma T^{T}} \sigma_{t} / a$, so $x^{\sigma^{T} \sigma_{t}{ }^{2} / a=x}$ or $x^{\sigma T_{B}} \sigma(x-a)=a(x-c)$. However computing this equation at $(a, 0)$ or $(c, 0)$ yields a contradiction. Let $\sqrt{d} \eta=\omega / t$. Then $\eta^{\sigma^{T} T_{\sigma}}=\eta$ and $d \eta^{2}=2\left(u^{2}+d v^{2}\right)+2(a-2 c)$. Combining these equations shows that $d \eta^{2}=2\left(2 u^{2}-a\right)+2(a-2 c)=4 u^{2}-4 c$. Thus we have proved

Proposition 5.5. With notation as above, $K_{11}$ is described by $u^{2}-d v^{2}=$ a while $K_{1}$ is described by the pair of equations $u^{2}-d v^{2}=a$ and $(2 u)^{2}-$ $d \eta^{2}=4 c$.

As a simple example of these techniques, consider the equation $2 \omega^{2}=$ $4 t^{4}+36 t^{2}+9$ or, equivalently, $\omega^{2}-2\left(t^{2}+(9 / 2)\right)^{2}=-36$. This has no points in $Q$. Indeed its Jacobian is $y^{2}=x(x-3)(x-6)$ and 3 is not a norm from $Q(\sqrt{2})$.

\section{BIBLIOGRAPHY}

1. J. W. S. Cassels, Diophantine equations with special reference to elliptic curves, J. London Math. Soc. 41 (1966), 193-291; corrigenda, ibid. 42 (1967), 183. MR 33 \#7299; 34 \#2523.

2. M. Eichler, Einführung in die Theorie der algebraischen Zahlen und Funktionen, Lehrbücher und Monographien aus dem Gebiete der exakten Wissenschaften, Mathematische Reihe, Band 27, Birkhäuser Verlag, Basel-Stuttgart, 1963; English translo, Pure and Appl. Matho, vol. 23, Academic Press, New York, 1966. MR 29 \#5821; $35 \# 160$.

3. R. E. MacRae dnd P. Samuel, Subfields of index 2 of elliptic function fields, Lecture Notes in Matho, vol. 311, Springer-Verlag, New York, 1972, pp. 171-193.

4. G. Poitou, Dans les pas de François Chatalet, Journées Arithmétiques, 1965, Faculté des Science de Besancon.

DEPARTMENT OF MATHEMATICS, UNIVERSITY OF COLORADO, BOULDER, COLORADO 80302 (Current address) FRANCE

DÉPARTEMENT DE MATHÉMATIQUE, UNIVERSITÉ DE PARIS, SUD XI, ORSAY, 